\title{
Dual-wavelength vertical external-cavity surface-emitting laser: strict growth control and scalable design
}

\author{
Agata Jasik $^{1}$ - Adam Kacper Sokót ${ }^{2}$ - Artur Broda ${ }^{1} \cdot$ Iwona Sankowska $^{1}$ • \\ Anna Wójcik-Jedlińska ${ }^{1}$ Michał Wasiak ${ }^{2} \cdot$ Justyna Kubacka-Traczyk $^{1}$. \\ Jan Muszalski $^{1}$
}

Received: 30 September 2015 / Accepted: 7 December 2015 / Published online: 27 January 2016

(c) The Author(s) 2016. This article is published with open access at Springerlink.com

\begin{abstract}
This paper reports on the design and fabrication of a dual-wavelength vertical external-cavity surfaceemitting laser. Grown by molecular beam epitaxy, the laser structures have a relatively simple active region divided into two sections, between which there is no optical filter. Comparable threshold power was achieved for both wavelengths. The growth rate was controlled precisely by growing $\mathrm{AlAs} / \mathrm{GaAs}$ superlattices with different period thicknesses and testing them with high-resolution X-ray diffractometry. The simultaneous emission of two wavelengths was detected in setup without a heat spreader, one of $991 \mathrm{~nm}$ and the other of $1038 \mathrm{~nm}$. After diamond heat spreader was bonded, both wavelengths lased in continuous-wave mode with the combined output power of $1.79 \mathrm{~W}$. The design scalability allowed us to obtain two further structures with layers thinned by about $3 \%$ in the first and by about $6 \%$ in the second, operating at 958/1011 and $928 / 977 \mathrm{~nm}$, respectively.
\end{abstract}

\section{Introduction}

Vertical external-cavity surface-emitting lasers (VECSELs) are of great interest because of their circular neardiffraction limited beams and high output power [1, 2]. Their applications range from intracavity nonlinear frequency mixing [3-5] to two-wavelength interferometry

Agata Jasik

ajasik@ite.waw.pl

1 Institute of Electron Technology, Al.Lotników 32/46, 02-668 Warsaw, Poland

2 Institute of Physics, Łódź University of Technology, ul. Wólczańska 219, 90-924 Lodz, Poland
[6]. Several approaches have already been demonstrated to achieve dual-wavelength lasing. The frequently used approach is to apply an array of lasers with separated gain media for each wavelength $[7,8]$. However, this solution is quite bulky and requires complex optics. Some more compact devices have vertical coupled-cavity geometry $[9,10]$. In such cases, however, it is not possible to obtain larger separation between wavelengths of emitted radiation [11]. Generally, the most preferable laser is a monolithic source generating two coaxial beams simultaneously in the same optical cavity. Morozov et al. [12] proposed a theoretical model for such a laser in 2004. Shortly afterward, simultaneous dual-wavelength emission from an optically pumped VECSEL was demonstrated for the first time [13]. Uniform concentrations of carriers in quantum wells (QWs), located in the active region, were achieved by dividing the gain region into three sections, a long-wavelength section placed between two short-wavelength sections [13, 14]. To prevent interaction between the fields, the deep wells that emit the long wavelength were located near the electric field nodes of short-wavelength radiation [13-15]. In alternative designs, a filter was introduced between regions of shallow and deep wells $[16,17]$. Both structures are highly complex and difficult to implement. Three-sectional design requires either two indium sources or strict control of the In streams to provide InGaAs wells of different chemical composition. Structures with optical filters are much thicker and require very high long-term stability during the growing process. Even for much simpler nanostructures, very accurate control of the growth rate (better than $1 \%$ ) is usually required. For these reasons, reduction in the thickness of dual-wavelength VECSEL is preferable. It can be achieved by, for instance, using a two-sectional gain region without any filter, what was theoretically proposed by Yu.A.Morozow. [18] 
In this paper, we present experimentally performed a simpler geometry for optically pumped dual-wavelength VECSELs than earlier reported designs, [12-20] which provides comparable threshold power for both wavelengths maintaining the value of output power on reported level. Ex situ growth rate control enabled us to create laser structures consistent with the design. The simplicity of the design and strict growth control allowed us to use the scalability of the laser structure to obtain spectral tuning over the range of $1040 \mathrm{~nm}$ (limited by the long wavelength) $\div 930 \mathrm{~nm}$ (limited by the short wavelength).

\section{Design of the laser structure}

Designing a dual-wavelength vertical external-cavity surface-emitting laser (DW VECSEL) is much more complicated than designing a typical VECSEL structure. The optical design is crucial in such devices, since it is necessary to consider the field distributions of two different waves. Moreover, to provide lasing at two different wavelengths, carriers generated in the active region have to be distributed properly among QWs for each wavelength. The exponential decay of the pumping beam requires a specific arrangement of QWs. Since many of the optical, diffusion and gain parameters are temperature dependent, it is also necessary to know the exact temperature distribution within the semiconductor structure. To analyze the optical properties of our structure, we used the effective frequency method (EFM) $[21,22]$. To find the optimal design of the active region, we applied the 1-dimensional carrier transport model [23]. The material gain of the QWs was calculated with the aid of the model described in Ref. [19]. A 3-dimensional heat transfer model utilizing the finite element method (FEM) was used for the thermal calculations [21, 24]. The overall structure of our dual-wavelength VECSEL is typical for a VECSEL, except in that the gain region has been designed for dual-wavelength emission. The detailed information about chemical compositions and thicknesses of all the layers in DW VECSEL structure is given in Table 1.

The distributed Bragg reflector (DBR) is made up of 29.5 pairs of alternating GaAs and AlAs layers, $\lambda / 4$ in thickness. The structure is completed by a window layer of AlAs capped by a 10-nm GaAs layer. The wavelengths emitted are approximately 980 and $1030 \mathrm{~nm}$. Figure 1 presents the refractive index and optical field intensities of our DW VECSEL.

The optical lengths of the micro-cavity are $8.5 \times \lambda_{\mathrm{S}}$ and $8.0 \times \lambda_{\mathrm{L}}$ for the short and long wavelengths, respectively. The active region is divided into two sections by a widebandgap blocking layer. The requirement for equal absorption of pump radiation in both sections and the assumption of exponential decay of pump radiation in the absorbing region shortened the length of Section I. To distribute the same number of QWs in both sections, the shallow QWs had to be paired. Section I, located close to the surface of the device, therefore contains three pairs of $\operatorname{In}_{0.18} \mathrm{Ga}_{0.82} \mathrm{As}$ QWs emitting at $\lambda_{\mathrm{S}}=980 \mathrm{~nm}$ (short-wavelength radiation $\lambda_{\mathrm{S}}$ from shallow $\mathrm{QW} \mathrm{W}_{\mathrm{S}}$ ). Section II, situated deeper in the structure, consists of six single QWs of $\operatorname{In}_{0.23} \mathrm{Ga}_{0.77}$ As emitting at $\lambda_{\mathrm{L}}=1026 \mathrm{~nm}$ (long-wavelength radiation $\lambda_{\mathrm{L}}$ from deep $\mathrm{QW}_{\mathrm{L}}$ ).

All the QWs were placed near the antinodes of the corresponding standing wave to enhance the gain coefficient. To minimize the optical absorption of short-wavelength emissions in the deep QWs, the $\mathrm{QW}_{\mathrm{L}} \mathrm{s}$ were placed near
Table 1 The epistructure of DW VECSEL

\begin{tabular}{|c|c|c|c|c|c|c|c|}
\hline No & Layer & Material & Thickness $(\mu \mathrm{m})$ & No & Layer & Material & Thickness $(\mu \mathrm{m})$ \\
\hline 1 & Cap & GaAs & 0.01 & 17 & Spacer & GaAs & 0.429 \\
\hline 2 & Window & AlAs & 0.158 & 18 & QW & $\mathrm{In}_{0.23} \mathrm{Ga}_{0.77} \mathrm{As}$ & 0.008 \\
\hline 3 & Spacer & GaAs & 0.12 & 19 & Spacer & GaAs & 0.13 \\
\hline 4 & QW & $\mathrm{In}_{0.18} \mathrm{Ga}_{0.82} \mathrm{As}$ & 0.008 & 20 & QW & $\mathrm{In}_{0.23} \mathrm{Ga}_{0.77} \mathrm{As}$ & 0.008 \\
\hline 5 & Spacer & GaAs & 0.01 & 21 & Spacer & GaAs & 0.13 \\
\hline 6 & QW & $\mathrm{In}_{0.18} \mathrm{Ga}_{0.82} \mathrm{As}$ & 0.008 & 22 & QW & $\mathrm{In}_{0.23} \mathrm{Ga}_{0.77} \mathrm{As}$ & 0.008 \\
\hline 7 & Spacer & GaAs & 0.112 & 23 & Spacer & GaAs & 0.13 \\
\hline 8 & QW & $\mathrm{In}_{0.18} \mathrm{Ga}_{0.82} \mathrm{As}$ & 0.008 & 24 & QW & $\mathrm{In}_{0.23} \mathrm{Ga}_{0.77} \mathrm{As}$ & 0.008 \\
\hline 9 & Spacer & GaAs & 0.01 & 25 & Spacer & GaAs & 0.13 \\
\hline 10 & QW & $\mathrm{In}_{0.18} \mathrm{Ga}_{0.82} \mathrm{As}$ & 0.008 & 26 & QW & $\mathrm{In}_{0.23} \mathrm{Ga}_{0.77} \mathrm{As}$ & 0.008 \\
\hline 11 & Spacer & GaAs & 0.112 & 27 & Spacer & GaAs & 0.13 \\
\hline 12 & QW & $\mathrm{In}_{0.18} \mathrm{Ga}_{0.82} \mathrm{As}$ & 0.008 & 28 & QW & $\mathrm{In}_{0.23} \mathrm{Ga}_{0.77} \mathrm{As}$ & 0.008 \\
\hline 13 & Spacer & GaAs & 0.01 & 29 & Spacer & GaAs & 0.48 \\
\hline 14 & QW & $\mathrm{In}_{0.18} \mathrm{Ga}_{0.82} \mathrm{As}$ & 0.008 & 30 & DBR 1 & AlAs & 0.0840 \\
\hline 15 & Spacer & GaAs & 0.154 & 31 & DBR 2 & GaAs & 0.0710 \\
\hline 16 & Block & AlAs & 0.03 & \multicolumn{4}{|c|}{29.5 pairs of $\mathrm{DBR}$} \\
\hline
\end{tabular}




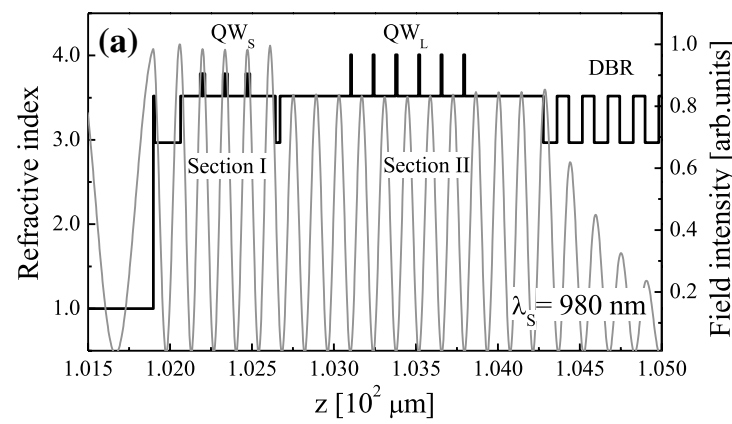

Fig. 1 The refractive index (in black) and optical field intensities (in gray) at $\lambda_{\mathrm{S}}=980 \mathrm{~nm}(\mathbf{a})$ and $\lambda_{\mathrm{L}}=1026 \mathrm{~nm}$ (b) for a dual-wavelength VECSEL. $Q W_{S}$ quantum well emitting short-wavelength radia-

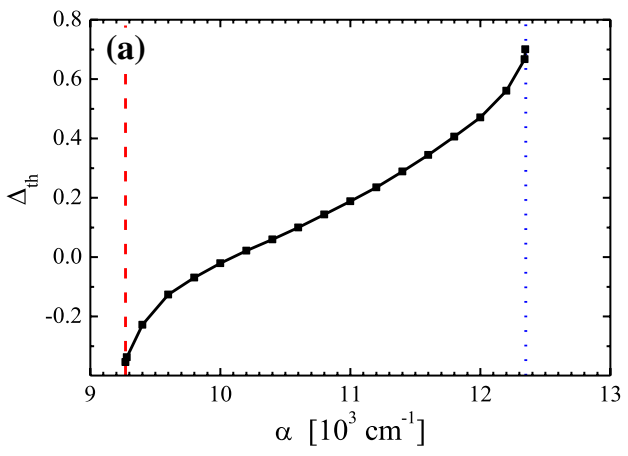

Fig. 2 The relative threshold separation [see Eq. (1)] versus absorption (a) and diffusion coefficients (b) in GaAs. The following values are assumed: in a $D=10 \mathrm{~cm}^{2} / \mathrm{s}$ and in $\mathbf{b} \alpha=10^{4} \mathrm{~cm}^{-1}$. The same values were used in the actual design. In a the dashed (red) and dot-

the nodes of $\lambda_{S}$. For the $\lambda_{L}$, the $\mathrm{QW}_{\mathrm{S}} \mathrm{S}$ were only slightly shifted from the antinodes of the $\lambda_{\mathrm{L}}$ standing wave. However, thanks to their wider bandgap, the wavelength $\lambda_{\mathrm{L}}$ is hardly absorbed by the shallow QWs.

The blocking layer was made from $30 \mathrm{~nm} \mathrm{AlAs}$ and is transparent to the pump light and laser emissions, but impenetrable to charge carriers. It enables each section to be pumped independently and prevents the deep QWs from being filled up by carriers generated in the shallow QWs barriers. Otherwise, the $\mathrm{QW}_{\mathrm{L}} \mathrm{s}$ would become more populated than the shallow QWs and this would lead ultimately to only the long-wavelength lasing. The location of the blocking layer is of great importance. The $\mathrm{QW}_{\mathrm{L}} \mathrm{s}$ have longer carrier escape time and shorter capture time [15]; therefore, the blocking layer should promote the excitation of the shallow QWs. However, at the same time, it should not disturb long-wavelength radiation penetrating into Section II, as this could significantly deplete or even prevent emission at $\lambda_{\mathrm{L}}$. Therefore, the optimal position of the QWs and of the AlAs layer was determined numerically to achieve a comparable level of threshold power for both

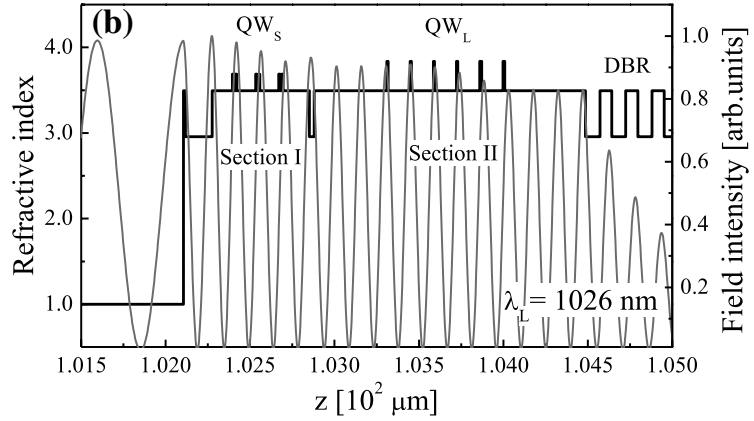

tion, shallow $\mathrm{QW}, Q W_{L}$ quantum well emitting long-wavelength radiation, deep QW

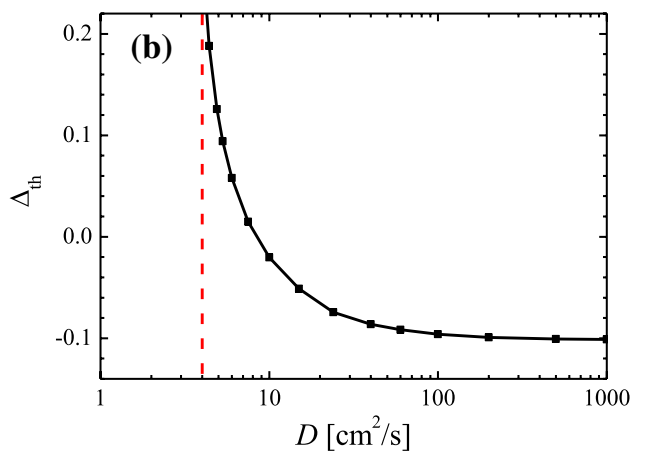

ted (blue) lines mark the limits of short- and long-wavelength emission, respectively. In between there is a region of dual-wavelength operation. In $\mathbf{b}$ the dashed line indicates the limit of the long-wavelength emission

emitted wavelengths. Moreover, our simulations show that the absorption coefficient $\alpha$ of GaAs at $810 \mathrm{~nm}$ (the pumping wavelength) and the diffusion coefficient $D$ in the active region are important parameters which strongly influence the emission characteristics, especially for the longer wavelength generated in Section II (see Fig. 1). Higher absorption of the pumping radiation means that more carriers are generated in Section I at the expense of Section II. Therefore, the threshold level of excitation for the shorter wavelength (generated in Section I) is a decreasing function of $\alpha$, while for the longer wavelength it is an increasing function of $\alpha$. In Fig. 2 we present the relative threshold separation as functions of absorption and diffusion coefficients in GaAs. The relative threshold separation is defined by the following formula:

$\Delta_{\mathrm{th}}=\frac{P_{\mathrm{th}}^{\mathrm{L}}-P_{\mathrm{th}}^{\mathrm{S}}}{\min \left(P_{\mathrm{th}}^{\mathrm{L}}, P_{\mathrm{th}}^{\mathrm{S}}\right)}$

where $P_{\mathrm{th}}^{\mathrm{L}}, P_{\mathrm{th}}^{\mathrm{S}}$ are threshold pumping powers for the long and short wavelengths, respectively. 
On the other hand, when diffusion is more efficient $(D$ is higher), the carrier distribution in Section II (which is almost three times thicker than in Section I) becomes more homogenous, increasing the total gain for the longer wavelength. This is why, as can be seen in Fig. 2b, when the diffusion constant is lower the shorter wavelength reaches its threshold before the longer wavelength reaches its threshold, whereas when $D$ is higher, the situation is reversed, and the longer wavelength reaches its threshold first. The threshold of the shorter wavelength is practically independent of the diffusion coefficient within the considered range.

The VECSEL described in this paper thus utilizes only two gain sections in the active region, has no optical filter and has comparable emission thresholds for short and long wavelengths. What distinguishes our structure from other reported DW VECSELs is the simple design. This simple design is easier to implement as there is no need for rigorous control of the In source or for long-term stability during the manufacturing process.

\section{Structure fabrication}

The test structures and DW VECSELs were grown by molecular beam epitaxy. A Riber 32P reactor was equipped with standard Knudsen effusion cells for group III elements and with a valved cracking cell for arsenic. The temperature was measured with an Ircon Modline pyrometer operating at $930 \mathrm{~nm}$. The growth temperature was $530{ }^{\circ} \mathrm{C}$ and kept constant during the whole epitaxial process. The growth rate was controlled in situ by reflection high-energy electron diffraction (RHEED) oscillations and ex situ by high-resolution X-ray diffractometry (HR XRD). Before the epitaxial growth of the DW VECSEL structures, the active regions containing two types of QW, the DBR structures and the VECSEL structures emitting separately short and long wavelengths were optimized.

As reported by Morozov et al. [15], a 6-nm shift from the position of each $\mathrm{QW}_{\mathrm{L}}$ causes instability in the lasing operation. This means that execution (i.e., laser structure design and fabrication) must be controlled to within $0.5 \%$. This is the limit value for the MBE technique fixed by flux instability in group III elements. Therefore, controlling the growth rate is a crucial technological issue.

The growth rate depends on the fluxes of the group III elements if all other technological parameters are kept constant. After opening the cells, a burst of fluxes is observed, following which the fluxes stabilize for about $3 \mathrm{~min}$ for $\mathrm{Ga}$ and 1.5 min for $\mathrm{Al}$ (see Fig. 3). As a consequence, the rates at which layers made from the same alloy grow a few nms or a few tens of nms differ significantly. Our DW VECSEL structure includes a thin GaAs layer of $10 \mathrm{~nm}$ thickness (the cap layer) and thick layers of GaAs of around

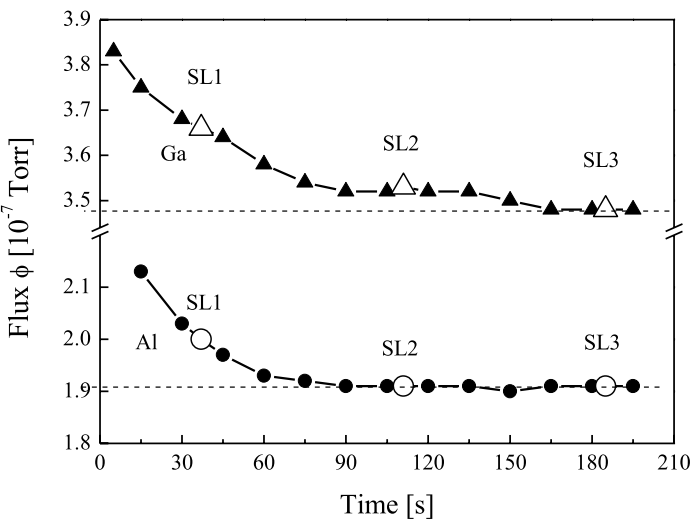

Fig. 3 Fluxes of group III elements. Open symbols indicate the growth parameters of 30-period AlAs/GaAs SLs

$130 \mathrm{~nm}$ (barrier layers), as well as a thin AlAs layer of $30 \mathrm{~nm}$ (the thin blocking layer) and thick AlAs layers of around $158 \mathrm{~nm}$ (window layer). This requires determining the growth rate of the thin and the thick layers separately.

The investigations were carried out using X-ray diffraction. Since single layers of a few $\mathrm{nm}$ in thickness are too thin for these studies, three periodic structures of $30 \times \mathrm{AlAs} /$ GaAs of various thicknesses were grown: SL1 with a period of 10/10 nm, SL2 with a 30/30 nm period and SL3 with a $50 / 50 \mathrm{~nm}$. The deposition times for the SL components were determined assuming a growth rate estimated from RHEED oscillations of $0.97 \mu \mathrm{m} / \mathrm{h}$ for both layers. The results of the HR XRD measurements are presented in Figs. 4 and 5.

The thicknesses of the binaries were obtained from simulations of experimental diffraction profiles (Fig. 4), following which the deviation of the growth rates for the thin layers $(10$ and $30 \mathrm{~nm})$ from the growth rate of thick layer $(50 \mathrm{~nm})$ was calculated. As can be seen in Fig. 5, AlAs material shows higher deviation in growth rates. The thinnest AlAs layer (SL1) shows the highest deviation at $7.1 \%$, while the higher deviation of $2.5 \%$ for GaAs layer (SL1) is comparable the lower deviation of the thicker AlAs layer (SL2) at $2.2 \%$.

The deposition times for all layers in the DW VECSEL structures were determined precisely, taking into account the dependence of the growth rate on layer thickness for both AlAs and GaAs binaries. As a result, a heterostructure consistent with the design was obtained. No anti-reflection coating was deposited on the VECSEL surface.

\section{Structure and laser evaluation}

The as-grown heterostructure was examined by photoluminescence and reflectance spectroscopy. The results are presented in Fig. 6. 


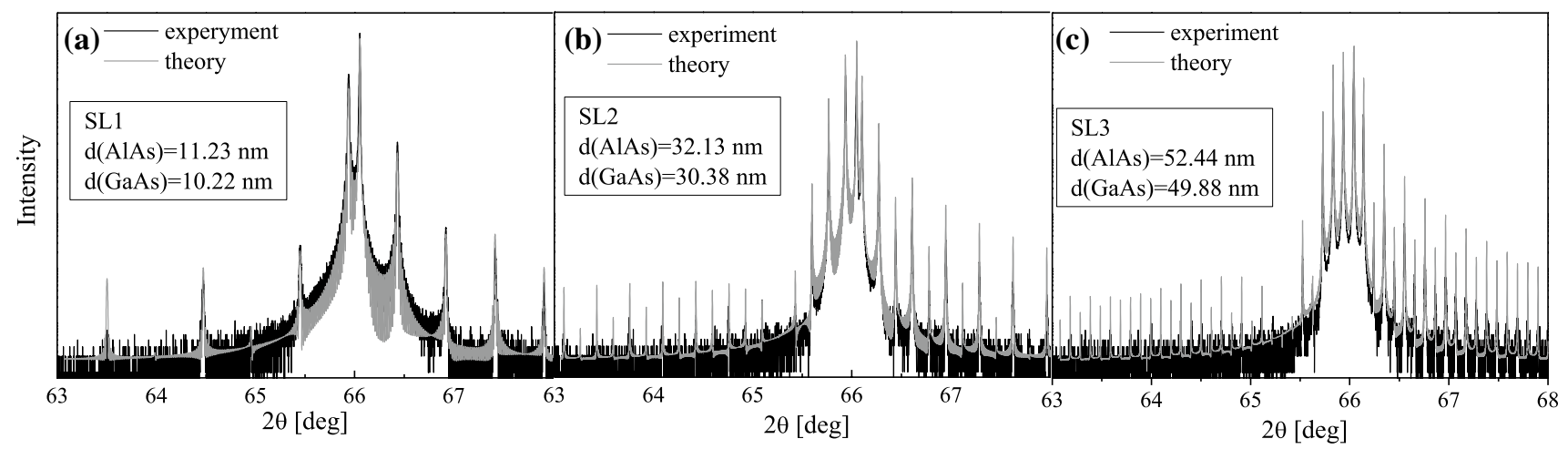

Fig. 4 HR XRD profiles measured for 30-period AlAs/GaAs SLs. Thicknesses are determined from simulations of the experimental profiles

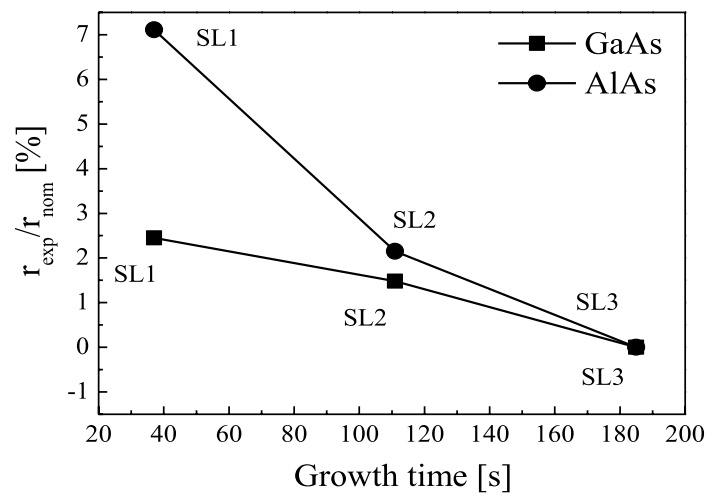

Fig. 5 Deviation growth rate of AlAs and GaAs layers

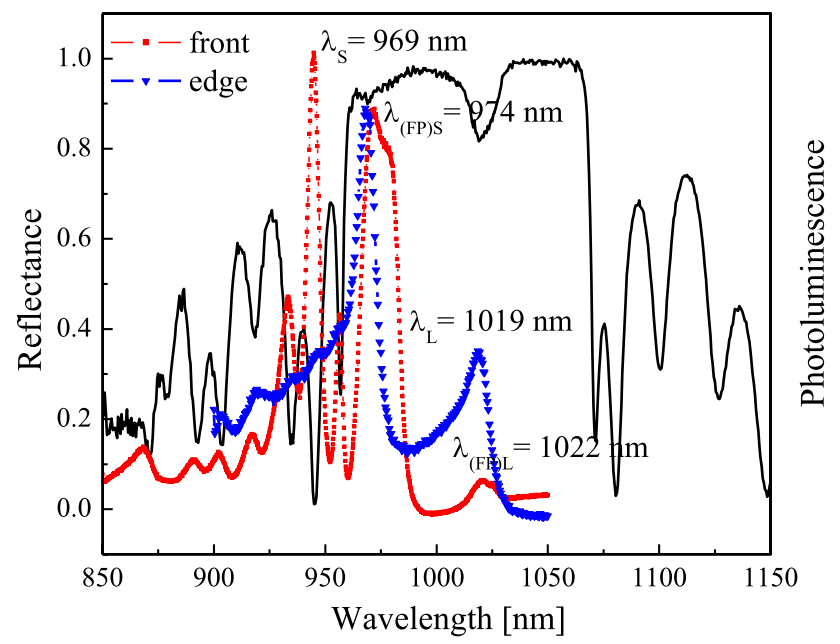

Fig. 6 The reflectance $R$ and PL spectra for the DW VECSEL. The symbols designate PL spectra taken from the surface (squares) and from the edge (triangles). $\lambda_{\mathrm{S}}=969$ and $\lambda_{\mathrm{L}}=1019 \mathrm{~nm}$-emission wavelengths originate from shallow and deep QWs, respectively. The F-P resonances are at wavelengths of $\lambda_{(\mathrm{F}-\mathrm{P}) \mathrm{S}}=974$ and $\lambda_{(\mathrm{F}-\mathrm{P})}$ $\mathrm{L}=1022 \mathrm{~nm}$
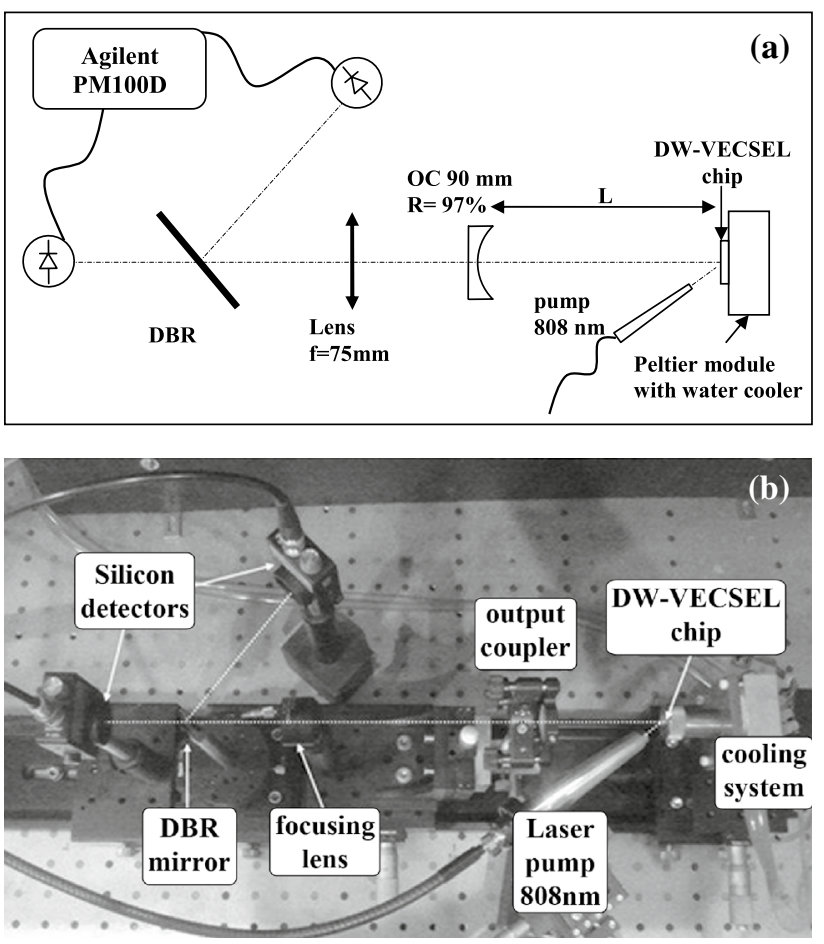

Fig. 7 The DW VECSEL cavity scheme for (a) and photograph of the setup (b)

The PL spectrum measured from the surface, and the dips in reflectance clearly indicate wavelengths of F-P resonances, which are $\lambda_{(\mathrm{F}-\mathrm{P}) \mathrm{S}}=974 \mathrm{~nm}$ and $\lambda_{(\mathrm{F}-\mathrm{P})}$ $\mathrm{L}=1022 \mathrm{~nm}$, whereas the PL spectrum taken from edge of the structure shows emission from shallow and deep QWs at wavelengths of $\lambda_{\mathrm{S}}=969 \mathrm{~nm}$ and $\lambda_{\mathrm{L}}=1019 \mathrm{~nm}$, respectively. This means that the structure was made with an accuracy of around $0.5 \%$, which is the limit for MBE processes. 
To evaluate the lasing properties, a cleaved $3 \times 3 \mathrm{~mm}^{2}$ chip was set directly into a linear resonator. The chip was mounted on a heat sink using a thermoconductive paste. The heat sink temperature was regulated by a Peltier module. The chip was kept at a range of constant temperatures between $-4 \div+10{ }^{\circ} \mathrm{C}$.

The resonator was formed using an integrated Bragg reflector and an external output coupler OC. The curvature of output coupler was $90 \mathrm{~mm}$, and its reflectivity was 97 and $98 \%$ for a laser chip without diamond heat spreader and for a bonded chip, respectively. The DW VECSEL was optically pumped by a fiber-coupled $800 \mathrm{~nm}$-laser diode. Since unprocessed wafers were used (i.e., no heat spreader, and without removing or thinning the substrate), to limit excessive heating of the heterostructure the diameter of the pump beam spot was limited to $60 \mu \mathrm{m}$. Although the small spot size limited the total output power, it permitted rapid assessment of the epitaxial material. The structure bonded to $300-\mu \mathrm{m}$-thick single crystal diamond inside copper holder was pumped with $200 \mu \mathrm{m}$ spot of light beam.

To examine the laser emission at each wavelength (988 and $1038 \mathrm{~nm}$ ) independently, the two components of the lasing beam were spatially separated and registered using two independent fast photo-detectors (DET10A, Thorlabs). In the experimental setup presented in Fig. 7 (in this case, for VECSEL without diamond $T=2 \%$ ), a spare DBR wafer was used as a wavelength tunable filter. At the angle of beam incidence of $32^{\circ}$, the DBR mirror has a high reflectance for the shorter laser wavelength of $991 \mathrm{~nm}$ (plateau) and a high transmission for the longer laser wavelength of $1038 \mathrm{~nm}$ (the first minimum). The DBR was inserted into the DW VECSEL output beam at an angle of $32^{\circ}$, separating the laser wavelengths by reflection and transmission. Figure 8 presents lasing spectra taken at $P_{\text {pump }}=700 \mathrm{~mW}$. Both wavelengths lased in continuous-wave mode. The wavelength spectral separation was as much as $47 \mathrm{~nm}$. Such large spectral separation is not achievable by any other intracavity technique. [25]

The reflected beam contains about $15 \%$ of the longer wavelength, but this is negligible in comparison with the intensity of the shorter wavelength (Fig. 8b). The transmitted and reflected beams are polarized in the same way. Linear polarization runs in [0-1-1] crystallographic direction with a contrast of 1000:1. This high contrast ratio indicates anisotropic strain relaxation due to the large number of QWs lattice-mismatched to the GaAs substrate.

Time-dependent measurements taken with a $500 \mathrm{MHz}$ oscilloscope show that lasing is simultaneous at both wavelengths, and there was no observable switching between wavelengths (as has been reported by other authors [13]).

Our goal was to make a laser with a common threshold for both wavelengths. Figure 9, which presents the output

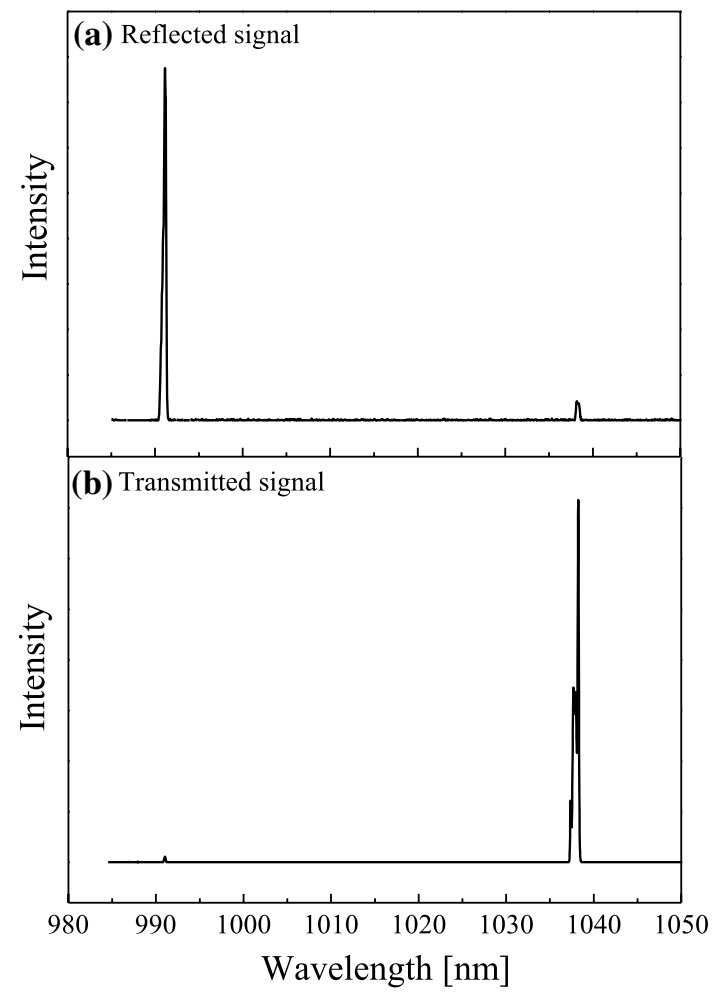

Fig. 8 Lasing spectral characteristics of DW VECSEL: transmitted (a) and reflected (b) spectral line. The output coupler: $R=90 \mathrm{~mm}$, $T=2 \%$. The temperature on the heat sink was $10^{\circ} \mathrm{C}$. The diameter of the pump beam spot was $60 \mu \mathrm{m}$

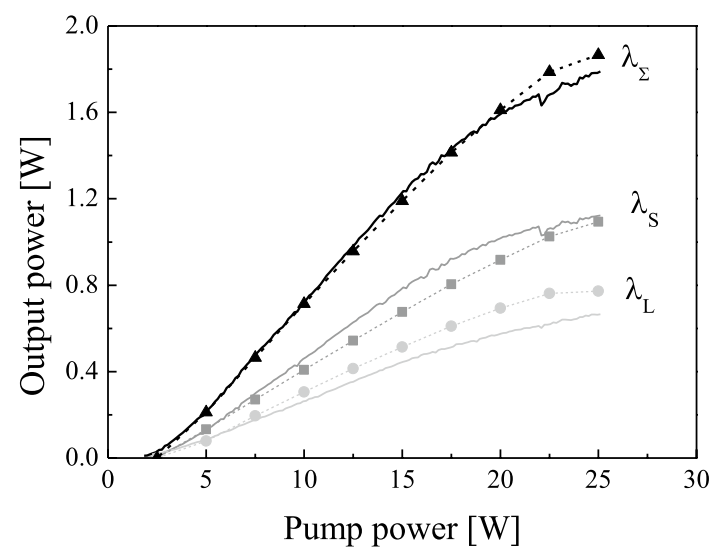

Fig. 9 Light output plotted against pump power for short and long wavelengths. The output coupler: $R=90 \mathrm{~mm}, T=3 \%$. The temperature on the heat sink was $-4{ }^{\circ} \mathrm{C}$. The diameter of the pump beam spot was $200 \mu \mathrm{m}$. Symbols—simulated data, lines — measured curves

power $P_{\text {out }}$ versus power of pump $P_{\text {in }}$, proves that we have managed to achieve this goal. However, it required not only taking into account the optical properties of the materials, but also analysis of the carrier transport in the structure, as shown in Fig. 2. 

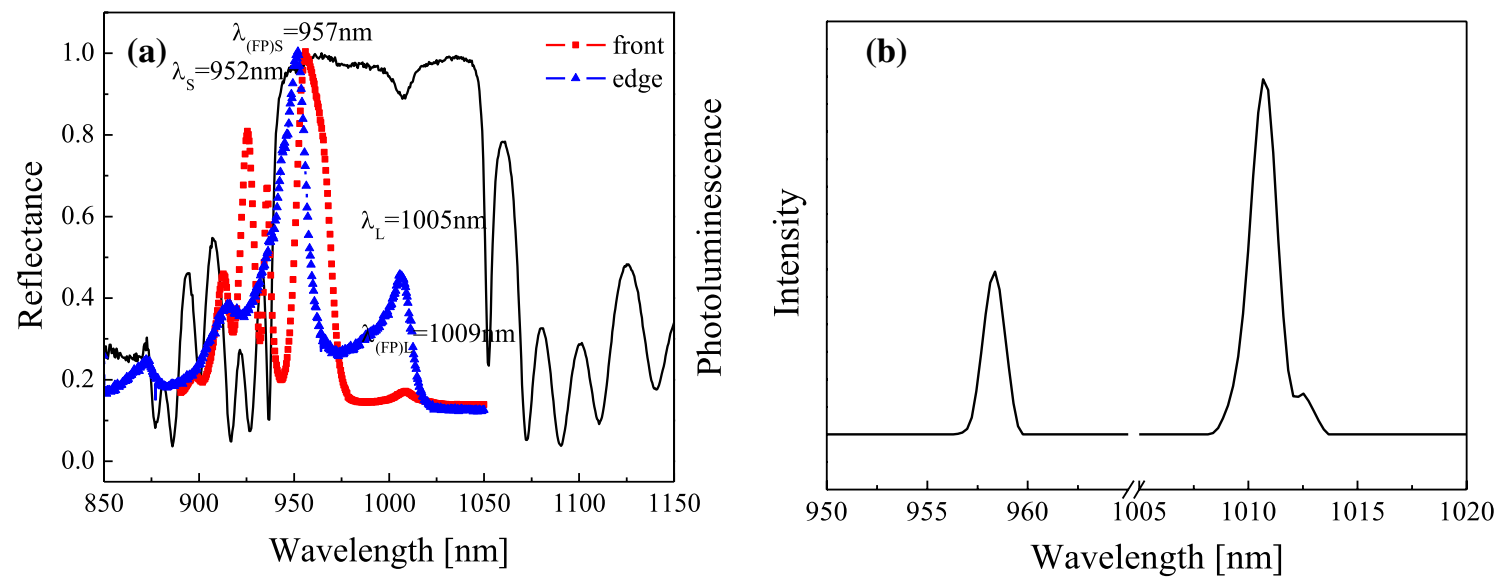

Fig. 10 The spectral characteristics of reflectance and photoluminescence (a) and the lasing spectrum (b) taken at $P_{\text {pump }}=0.5 \mathrm{~W}$ from a DW VECSEL thinned by $3 \%$. The output coupler: $R=90 \mathrm{~mm}, T=2 \%$. The temperature on the heat sink was $-5{ }^{\circ} \mathrm{C}$
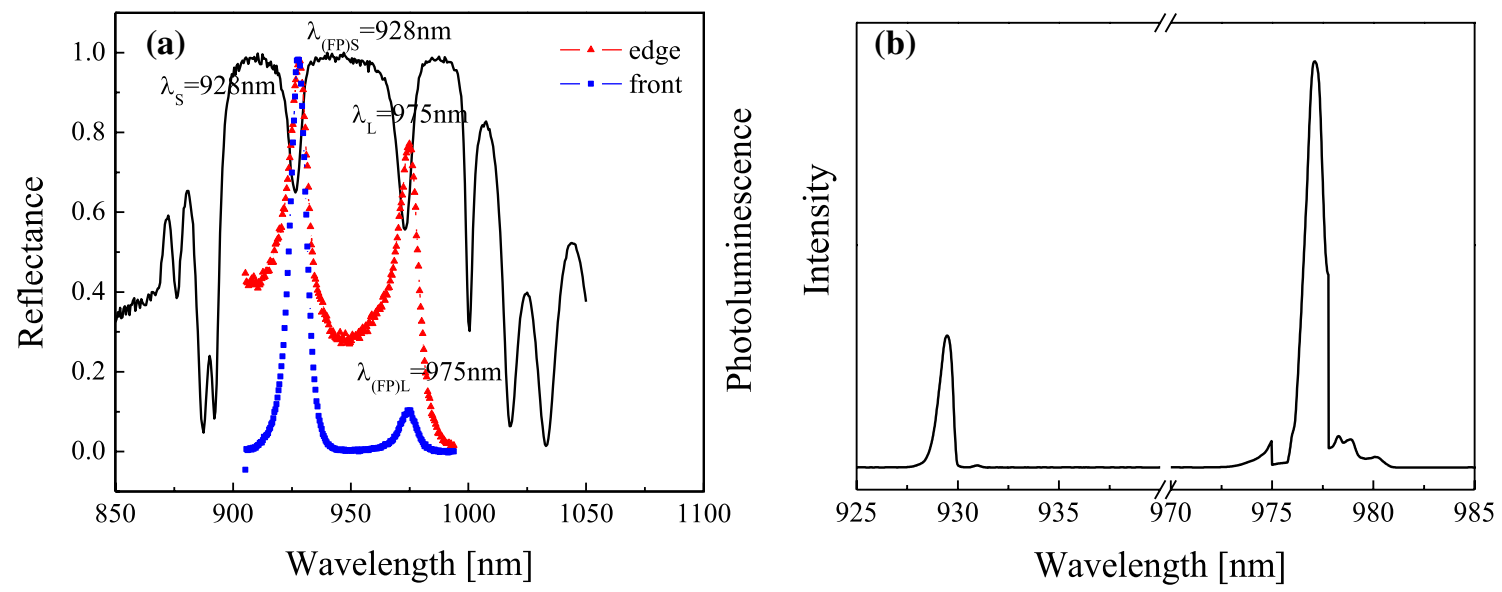

Fig. 11 The spectral characteristics of reflectance and photoluminescence (a) and the lasing spectrum (b) taken at $P_{\text {pump }}=0.5 \mathrm{~W}$ from a DW VECSEL thinned by $5 \%$. The output coupler: $R=90 \mathrm{~mm}, T=2 \%$. The temperature on the heat sink was $0{ }^{\circ} \mathrm{C}$

The laser generated $\mathrm{CW}$ optical power of $1.12 \mathrm{~W}$ at $\lambda_{\mathrm{S}}=977 \mathrm{~nm}$ and $0.67 \mathrm{~W}$ at $\lambda_{\mathrm{L}}=1024 \mathrm{~nm}$ at the pump power of $25 \mathrm{~W}$. The combined output power reached its maximum of about $1.79 \mathrm{~W}$. The roll-over of any characteristic was not observed up to the pump power of $25 \mathrm{~W}$. The good agreement between simulated and measured characteristics was obtained especially for the total output power.

\section{Spectral scalability}

The significant advantage of our approach, i.e., both the simplest design and technology precision is the possibility it presents for simple scalability. If the thickness of the micro-cavity is uniformly decreased and the QW emission is tuned to the new resonant conditions, then lasing performance can be maintained. According to this method, the original project was modified and two DW VECSEL structures were produced, thinned by about 3 and $6 \%$, respectively. The PL spectra, reflectance and lasing spectra of the modified heterostructures are presented in Figs. 10 and 11.

For the structure thinned by about $6 \%$ of its original width, lasing was observed at 928 and $977 \mathrm{~nm}$ and for the structure thinned by about $3 \%$, lasing occurred at 958 and $1011 \mathrm{~nm}$. It can be assumed that the DW VECSEL is scalable between the range of $0 \div 6 \%$. On the other hand, increasing the layer thickness in the DW VECSEL structure causes the lasing parameters to deteriorate due to increased strain relaxation in the active region. 


\section{Conclusion}

The DW VECSEL presented in this paper is, to the best of our knowledge, the simplest such structure yet produced. Careful design allowed us to obtain simultaneous emission and the same threshold for both wavelengths. We believe that by combining the DW VECSEL with a nonlinear crystal for difference-frequency generation (DFG), its relatively simple design, well-controlled growth rate and scalability could contribute to the development of new mid-IR $(5-50 \mu \mathrm{m})$ radiation sources.

Acknowledgments This work was partially supported by the National Science Centre (NCN) under project 3606/B/T02/2009/36, and by the National Centre for Research and Development (NCBiR) under projects 02-0009-10/2011 and 02-0023-06.

Open Access This article is distributed under the terms of the Creative Commons Attribution 4.0 International License (http://creativecommons.org/licenses/by/4.0/), which permits unrestricted use, distribution, and reproduction in any medium, provided you give appropriate credit to the original author(s) and the source, provide a link to the Creative Commons license, and indicate if changes were made.

\section{References}

1. M. Kuznetsov, F. Hakimi, R. Sprague, A. Mooradian, Highpower $(>0.5-\mathrm{W} \mathrm{CW}$ ) diode-pumped vertical-external-cavity surface-emitting semiconductor lasers with circular TEM00 beams. IEEE Photonics. Technol. Lett. 9(8), 1063-1065 (1997)

2. T.-L. Wang, B. Heinen, J. Hader, C. Dineen, M. Sparenberg, A. Weber, B. Kunert, S.W. Koch, J.V. Moloney, M. Koch, W. Stolz, Quantum design strategy pushes high-power vertical externalcavity surface-emitting lasers beyond $100 \mathrm{~W}$. Laser Photonics Rev. 6(5), L12-L14 (2012)

3. A. Härkönen, J. Rautiainen, T. Leinonen, Y.A. Morozov, L. Orsila, M. Guina, M. Pessa, O.G. Okhotnikov, Intracavity sumfrequency generation in dual-wavelength semiconductor disk laser. IEEE Photonics Technol. Lett. 19(19), 1550-1552 (2007)

4. C.L. Wang, C.L. Pan, Tunable multiterahertz beat signal generation from a two-wavelength laser-diode array. Opt. Lett. 20, 1767-1770 (1995)

5. M. Scheller, J.M. Yarborough, J.V. Moloney, M. Fallahi, M. Koch, S.W. Koch, Room temperature continuous wave milliwatt terahertz source. Opt. Express 18(26), 27112-27117 (2010)

6. C.L. Wang, Y.H. Chuang, C.L. Pan, Two-wavelength interferometer based on a two-color laser diode array and the second-order correlation technique. Opt. Lett. 20, 1071-1073 (1995)

7. C.L. Wang, C.L. Pan, Tunable multiterahertz beat signal generation from a two wavelength laser-diode array. Opt. Lett. 20, 1767-1770 (1995)

8. K.R. Poguntke, J.B.D. Soole, A. Scherer, H.P. LeBlanc, C. Caneau, R. Bhat, M.A. Koza, Simultaneous multiple wavelength operation of a multistrip array grating integrated cavity laser. Appl. Phys. Lett. 62, 2024-2026 (1993)
9. P. Michler, M. Hilpert, G. Reiner, Dynamics of dual-wavelength emission from a coupled semiconductor microcavity laser. Appl. Phys. Lett. 70, 2073-2075 (1997)

10. D.M. Grasso, K.D. Choquette, Threshold and modal characteristics of composite-resonator vertical-cavity lasers. IEEE J. Quantum Electron. 39(12), 1526-1530 (2003)

11. V. Badilita, J.-F. Carlin, M. Ilegems, K. Panajotov, Rate-equation model for coupled-cavity surface-emitting lasers. IEEE J. Quantum Electron. 40(12), 1646-1656 (2004)

12. Y. Morozov, I. Nefedov, V. Aleshkin, Nonlinear frequency conversion in a double vertical-cavity surface-emitting laser. Semiconductors 38(11), 1350-1355 (2004)

13. T. Leinonen, Y.A. Morozov, A. Harkonen, M. Pessa, Vertical external-cavity surface-emitting laser for dual-wavelength generation. IEEE Photonics Technol. Lett. 17(12), 2508-2510 (2005)

14. Y.A. Morozov, T. Leinonen, A. Härkönen, M. Pessa, Simultaneous dual-wavelength emission from vertical external-cavity surface-emitting laser: a numerical modeling. IEEE J. Quantum Electron. 42(10), 1055-1061 (2006)

15. Y.A. Morozov, I.S. Nefedov, T. Leinonen, M.Y. Morozov, Nonlinear-optical frequency conversion in a dual-wavelength vertical-external-cavity surface-emitting laser. Semiconductors 42(4), 463-469 (2008)

16. T. Leinonen, S. Ranta, A. Laakso, Y.A. Morozov, M. Saarinen, M. Pessa, Dual-wavelength generation by vertical external cavity surface-emitting laser. Opt. Express 15(20), 13451-13456 (2007)

17. M.Y. Morozov, Y.A. Morozov, V.V. Popov, Effect of pump wave reflections on the excitation of a dual-wavelength vertical-cavity surface-emitting laser. Semiconductors 43(3), 382-386 (2009)

18. Y.A. Morozov, A.I. Konyukhov, L.A. Kochkurov, M.Y. Morozov, Spatio-temporal dynamics of a dual-wavelength vertical-external-cavity surface-emitting semiconductor laser. Quantum Electron. 41(11), 1040-1044 (2011)

19. M.Y. Morozov, Y.A. Morozov, V.V. Popov, Features of dual wavelength generation in a vertical external cavity surface emitting laser. Tech. Phys. Lett. 36(4), 344-347 (2010)

20. Y.A. Morozov, M.Y. Morozov, I.V. Krasnikova, Pulsed difference frequency generation regime in dual wavelength vertical external cavity surface emitting laser. Tech. Phys. Lett. 37(12), 1112-1115 (2011)

21. Ł. Piskorski, M. Wasiak, R.P. Sarzała, W. Nakwaski, Tuning effects in optimisation of GaAs-based InGaAs/GaAs quantumdot VCSELs. Opt. Commun. 281(11), 3163-3170 (2008)

22. Ł. Piskorski, R.P. Sarzała, W. Nakwaski, Self-consistent model of $650 \mathrm{~nm}$ GaInP/AlGaInP quantum-well vertical-cavity surfaceemitting diode lasers. Semicond. Sci. Technol. 22(6), 593-600 (2007)

23. A.K. Sokół, R.P. Sarzała, Numerical analysis of optically pumped VECSELs. Proc. SPIE 8432, 84321H (2012)

24. T. Czyszanowski, R.P. Sarzała, Ł. Piskorski, M. Dems, M. Wasiak, W. Nakwaski, K. Panajotov, Comparison of usability of oxide apertures and photonic crystals used to create radial optical confinements in 650-nm GaInP VCSELs. IEEE J. Quantum Electron. 43(11), 1041-1047 (2007)

25. L. Fan, M. Fallahi, J. Hader, A.R. Zakharian, J.V. Moloney, W. Stolz, S.W. Koch, R. Bedford, J.T. Murray, Linearly polarized dual-wavelength vertical-external-cavity surface-emitting laser. Appl. Phys. Lett. 90, 181124 (2007) 